\title{
Comparative analysis of ESD versus EMR in a large European series of non-ampullary superficial duodenal tumors*
}

\section{(ㄷ)(1) $\odot($}

Authors

Enrique Pérez-Cuadrado-Robles ${ }^{1}$, Lucille Quénéhervé ${ }^{2}$, Walter Margos ${ }^{1}$, Leila Shaza ${ }^{1}$, Hrvoje Ivekovic ${ }^{1}$, Tom G. Moreels ${ }^{1}$, Ralph Yeung ${ }^{1}$, Hubert Piessevaux ${ }^{1}$, Emmanuel Coron ${ }^{2}$, Anne Jouret-Mourin ${ }^{3}$, Pierre H. Deprez ${ }^{1}$

Institutions

1 Department of Hepato-Gastroenterology, Cliniques universitaires Saint-Luc, Université Catholique de Louvain, Brussels, Belgium

2 Institut des Maladies de l'Appareil digestif, University Hospital of Nantes, France

3 Department of Pathology, Cliniques universitaires SaintLuc, Université Catholique de Louvain, Brussels, Belgium

Bibliography

DOI https://doi.org/10.1055/a-0577-7546 |

Endoscopy International Open 2018; 06: E1008-E1014

(c) Georg Thieme Verlag KG Stuttgart · New York

ISSN 2364-3722

Corresponding author

Pierre H. Deprez, Department of Hepato-Gastroenterology, Cliniques universitaires Saint-Luc, Université Catholique de Louvain, Brussels, Belgium

pdeprez@uclouvain.be

\section{ABSTRACT}

Background and study aims The choice of endoscopic submucosal dissection (ESD) or endoscopic mucosal resection (EMR) in non-ampullary superficial duodenal tumors (NASDTs) is challenging and the benefits of ESD remain unclear. The aim was to comparatively analyze the feasibility, outcomes and safety of these techniques in these lesions.

Patients and methods This is an observational and retrospective study. All consecutive patients presenting with
NASDTs who underwent EMR or ESD between 2005 and 2017 were included. The following main outcomes were comparatively evaluated: en-bloc and complete (R0) resection rates, and local recurrence. Secondary outcomes were perforation and delayed bleeding.

Results One hundred sixty-six tumors in 150 patients (age: 66 years, range: $31-83,42.7 \%$ males) were resected by $\operatorname{ESD}(n=37)$ or EMR $(n=129)$ and included. The median procedure time ( 81 vs. $50 \mathrm{~min}, P=0.007$ ) and tumor size ( $25 \mathrm{vs.}$ $20 \mathrm{~mm}, P=0.01$ ) were higher in the ESD group. The global malignancy rate was $50.3 \%$. There were no differences in en-bloc resection ( $29.7 \%$ vs. $44.2 \%, P=0.115)$, complete resection ( $19.4 \%$ vs. $35.5 \%, P=0.069$ ), and local recurrence ( $14.7 \%$ vs. $16.7 \%, P=0.788)$ rates. Tumor size was associated with recurrence ( 28 vs. $20 \mathrm{~mm}, P=0.008$ ), with a median follow-up of 6.5 months. Focal recurrence $(n=22,13.3 \%$ ) was treated endoscopically in $86.4 \%$. En-bloc resection in the ESD group was comparable in large $(\geq 20 \mathrm{~mm})$ and small lesions ( $27.6 \%$ vs. $37.5 \%, P=0.587)$, while this outcome decreased significantly in large lesions resected by EMR $(17.4 \%$ vs. $75 \%, P<0.001)$. Nine perforations were confirmed in 6 lesions (16.2\%) resected by ESD and 3 ( $2.3 \%)$ by EMR $(P=0.001)$. Endoscopic therapy was successful in all but 1 patient (88.9\%) presenting with a delayed perforation.

Conclusions ESD may be an alternative to EMR and surgery in selected NASDTs, such as large duodenal tumors where EMR achieves low en-bloc resection rates and the local recurrence may be higher. However, this technique may have a higher risk of perforations.

\section{Introduction}

Endoscopic submucosal dissection (ESD) has been described to achieve higher en-bloc resection and lower recurrence rates in esophageal, gastric, and colorectal tumors [1,2] when compar-

\footnotetext{
* Author note Enrique Pérez-Cuadrado-Robles, Lucille Quénéhervé and Walter Margos equally contributed to the conception and design and drafting of the article.
}

ed to endoscopic mucosal resection (EMR). This technique using knives can lead to complete resections in lesions with presumed submucosal invasion in preoperative diagnosis and avoid major surgery.

However, the duodenum represents a differentiated location with its own characteristics that can influence the outcomes of ESD in non-ampullary superficial duodenal tumors (NASDTs). The thin wall, narrow lumen, limited maneuverability, and stability of the scope and increased risk of complica- 
tions may lead to poor outcomes even in experienced referral centers. Additionally, the malignancy of NASDTs may be extremely difficult to determine based on the macroscopic characteristics [3]. Indeed, poor mucosal lifting may be due to the presence of Brunner's glands in the submucosal layer and not to fibrosis of invasive patterns.

Epithelial duodenal tumors are rare, and EMR has been proposed as the acceptable treatment in these cases, including large lesions requiring piecemeal approach [4]. Actually, there are no randomized controlled trials comparing ESD to EMR, and the retrospective published studies to date performing both techniques are based on different criteria [5-7]. Thus, the choice of ESD or EMR in these lesions is challenging and the benefits of ESD remain unclear. The aim of the present study was to comparatively analyze the feasibility, outcomes, and safety of these procedures in NASDTs.

\section{Methods}

\section{Patients}

This is an observational and retrospective study. All consecutive patients presenting with NASDTs who underwent ESD or EMR in our center between June 2005 and March 2017 were included. Pedunculated lesions or neuroendocrine tumors and lesions resected by polypectomy (without injection into submucosal layer) were not considered. Patients with ampullary tumors as well as those with a history of familial adenomatous polyposis were also excluded. Age, sex, tumor size, number of lesions, procedure time, endoscopic follow-up duration, and histological diagnosis were collected. The study protocol was approved by the local ethics committee and was registered as 2017/ $16 \mathrm{AOU} / 405$.

\section{EMR and ESD procedures}

Endoscopic resection approach was decided based on tumor size, macroscopic morphology, suspected type of lesion, location, and maneuverability. Previous endoscopic ultrasonography was not performed systematically. Basically, ESD was carried out when R0 was not supposed to be achieved by EMR, in case of local recurrence after previous endoscopic treatment, non-lifting sign, or suspicion of malignancy. Large superficial tumors were also intended to be resected by ESD. EMR was performed using a single-use symmetric (Captivator, Boston Scientific, Zaventem, Belgium) or asymmetric snare (Olympus, Berchem, Belgium) after submucosal injection by a mixture of epinephrine (dilution 1:50000), fluid gelatin and methylene blue, or normal saline injection. A forward-viewing gastroscope or side-viewing duodenoscope were used at the discretion of the endoscopist. ESD was performed by DualKnife (Olympus) as the main device, using monopolar current (VIO3/VIO300 D generators; ERBE, Zaventem, Belgium) by endocut I/Q (effect $1-2$ ) and forced/swift coagulation modes. Previous mucosal marking was carried out in large tumors or when the limits where unclear. A transparent hood was placed on the tip of the endoscope in all cases.

Hemostatic forceps (Coagrasper, Olympus) in soft coagulation mode (output: $80 \mathrm{~W} / 5.8$ ) was employed in cases of signifi- cant bleeding when DualKnife coagulation was insufficient. At the end of the excision, preventive hemostasis was decided on an individual basis and at the choice of the endoscopist. Prophylactic endoscopic closure to reduce the risk of delayed complications was performed using endoscopic clipping and/or endoloop (Olympus) if possible. The size of the excised lesion was measured after spreading onto a white cork surface, and tumor size was defined as the largest diameter. Radiological imaging was performed during the procedure in case of suspected perforation. All procedures were performed under general anesthesia by an experienced endoscopist (PD, HP, RY, TM) after written informed consent. A treatment by proton-pump inhibitors was proposed after resection in all cases, and a progressive liquid diet was indicated 12-48 hours after the procedure if there were no complications. Finally, a follow-up gastroscopy was indicated in $1-3$ months.

\section{Histopathological assessment}

The specimens were assessed by an expert pathologist (AJM). The resected specimen was cut into $2-3-\mathrm{mm}$ slices after fixation in formalin. Histological type, size, depth of invasion, lateral and vertical margins, and lymphovascular invasion were evaluated. Adenomatous tumors were classified into low-grade dysplasia, high-grade dysplasia, and adenocarcinoma. Histopathological grade was assigned according to the highest grade present in the tumor. Malignancy was defined as high-grade dysplasia or adenocarcinoma.

\section{Outcomes and definitions}

The following main outcomes were comparatively evaluated: en-bloc and complete (R0) resection rates and local recurrence. Secondary outcomes were delayed bleeding and perforation. En-bloc resection was defined as endoscopic resection of the tumor in 1 piece. Complete resection rate was only calculated in adenomatous/dysplastic NASDTs or carcinoma and was defined as resection of the tumor with free lateral and vertical margins. Delayed bleeding was defined as hematemesis or melena requiring endoscopic hemostasis, readmission, blood transfusion, or surgical intervention after the completion of the procedure. Perforation was classified as intraoperative or delayed as well as major or minor, based on whether intra-abdominal space was directly visualized. Local recurrence was defined as regrowth of adenomatous tissue at the site of the previous resection, confirmed by a positive histology during the follow-up. Follow-up endoscopy was routinely performed at 3 and 6 months following the resection and annually thereafter.

\section{Statistical analysis}

The main and secondary outcomes (categorical variables) were compared using $\mathrm{X}^{2}$ or Fisher's exact tests. A subgroup analysis including only tumors $\geq 20 \mathrm{~mm}$ resected by ESD or EMR was also carried out to highlight these outcomes in more comparable populations. Normally and non-normally distributed continuous variables were analyzed by Student's t-test and the Mann-Whitney U-test. They were presented as mean (SD) or median (range), respectively. A 2 -sided $P$-value of $<0.05$ was 
considered statistically significant. SPSS version 23 was used (IBM, SPSS Inc, Chicago, IL).

\section{Results}

\section{Patients}

Between 2005 and 2017, there were 203 patients presenting with non-ampullary neoplasms who underwent ESD or EMR. Those with familial adenomatous polyposis $(n=39)$ and subepithelial lesions ( $n=14$ ) were excluded. Thus, 166 NASDTs in 150 patients (age: 66 years, range: $31-83,42.7 \%$ males) were resected by ESD $(n=37)$ or EMR $(n=129)$ and were finally included. There were 2 patients with 3 tumors and 12 presenting with 2 lesions. Among these cases with multiple lesions, the endoscopic resection of all the tumors was performed during the same procedure in most of them $(n=11,78.6 \%)$.

Overall, most of the resected lesions (95.2\%) were located in D2 or distal with no differences between both groups (91.9\% vs. $96.1 \%, P=0.289)$. The median procedure time was higher in ESD cases ( 81 vs. $50 \mathrm{~min}, P=0.007$ ). The median tumor size was larger in lesions resected by ESD compared to EMR (25 vs. $20 \mathrm{~mm}, P=0.01$ ), while the overall endoscopic median size was $20 \mathrm{~mm}$ (range: 5 - 50). Indeed, 98 NASDTs (59\%) were $\geq 20 \mathrm{~mm}$ in size. Additionally, the $21.1 \%$ of tumors involved $\geq 50 \%$ of the duodenal circumference. The hybrid technique was used in most of ESD procedures $(n=29,78.4 \%)$ as shown in $>$ Fig. $\mathbf{1}$, and the cap-aspiration technique was used in 8 patients who underwent EMR.

\section{Comparative outcomes}

Concerning ESD/EMR outcomes, there were no differences in en-bloc and complete resection rates as shown in $>$ Table 1 . Incomplete resection in en-bloc resected lesions was due to positive lateral margins in all cases. Closure of the mucosal defect was carried out in $68.1 \%$ of patients with statistically significant differences between the first (2005 - 2011) and second periods $(2012-2017)$ of the study $(27.5 \%$ vs. $83.6 \%, P<0.001)$. Nine perforations were confirmed in 6 lesions resected by ESD and 3 by EMR $(P=0.001)$ ( $\$$ Table 2 ). All of them occurred during the first period of the study in tumors larger or equal to $20 \mathrm{~mm}$ (77.8\%) without prophylactic closure $(11.3 \%$ vs. $2.7 \%, P=$ $0.022)$. Most of the perforations in the ESD group occurred during full ESD technique ( $n=2 / 8,25 \%)$ followed by hybrid ESD approach $(n=4 / 39,13.8 \%)$. The median length of the hospitalization was 3 days (range: $2-15$ ). Notably, en-bloc resection was achieved in 3 lesions resected by ESD in spite of the perforation.

Overall, they were successfully treated during the procedure by standard $(n=7)$ clips, over-the-scope clips (Ovesco, Geetbets, Belgium) $(n=4)$, and endoloop (Olympus) $(n=2)$ in all but 1 patient who underwent emergency surgery after hybrid ESD. A 59-year-old woman underwent a piecemeal hybrid ESD for a 40-mm tumor with high-grade dysplasia located in D3. The mucosal defect was not closed due to the location of the lesion and limited scope maneuverability, but a contrast opacification performed after the procedure was normal. However, 12 hours later she presented with abdominal pain, and a delayed perforation was confirmed by CT abdominal scan, need- ing emergency open surgery. The patient was discharged after 14 days, and a feeding jejunostomy was placed for 40 days. Duodenal contrast opacification under radiological control was performed to ensure the closure of the intraoperative perforation in all cases and antibiotics were indicated.

The delayed bleeding rate was $9 \%$, with no differences between the ESD and EMR groups. Interestingly, two-thirds ( $\mathrm{n}=$ 10 ) of patients presenting with delayed bleeding did not have a mucosal defect closure following resection. Therefore, delayed bleeding was much more frequent in lesions without prophylactic closure ( $18.9 \%$ vs. $4.4 \%, P=0.002)$, and there was no bleeding in lesions located in the bulb.

\section{Histology and follow-up}

One hundred thirty-six patients (34 ESD, 102 EMR) underwent a median follow-up of 6.5 months (range: 2 -125). Local recurrence occurred in $5(14.7 \%)$ and $17(16.7 \%)$ tumors in the ESD and EMR groups, respectively $(P=0.788)$, and it was managed endoscopically in 19 patients and surgically in 3 cases. The global malignancy rate was $50.3 \%$ with comparable rates in the ESD and EMR groups (38.9\% vs. $56.7 \%, P=0.119)$. Similarly, the adenocarcinoma rate (median tumor size: $30 \mathrm{~mm}$ ) was comparable (5.6\% vs. $8.1 \%, P=0.607$ ). The median tumor size was higher in malignant lesions ( $25 \mathrm{~mm}$, range: 8 - 50) compared to benign tumors $(20 \mathrm{~mm}$, range: 5-50) $(P=0.001)$. Although malignancy was not statistically associated with local recurrence, adenocarcinomas presented a higher recurrence rate compared to adenomas with low-/high-grade dysplasia ( $36.4 \%$ vs. $15 \%, P=0.07$ ). Notably, all recurrent adenocarcinomas were resected by piecemeal EMR. Considering only patients with $\geq 12$ months follow-up $(n=60)$, the local recurrence rate was $23.3 \%$, with no statistically significant differences between the ESD $(n=5 / 11)$ and EMR groups $(n=9 / 49)(45.5 \%$ vs. $18.4 \%, P=0.055)$. However, overall tumor size was associated with recurrence ( 28 vs. $20 \mathrm{~mm}, P=0.008$ ). All the long-term recurrences in the ESD group underwent the hybrid technique, and most of tumors in the EMR group (88.8\%) were resected in piecemeal fashion. En-bloc ( $10.6 \%$ vs. $19.1 \%, P=0.202)$ and complete resection ( $12.1 \%$ vs. $18.6 \%, P=0.394$ ) were not statistically associated with lower recurrence rates in global analysis or subgroup analysis.

\section{Subgroup analysis}

A subgroup analysis of 98 lesions larger or equal than $20 \mathrm{~mm}$ (29 ESD, 69 EMR) was carried out. Thus, there were no differences in tumor size for both techniques $(P=0.711)$. The en-bloc (27.6\% vs. $17.4 \%, P=0.253$ ) and complete resection rates ( $13.8 \%$ vs. $11.6 \%, P=0.762$ ) were higher in the ESD group and the recurrence rate was lower in the ESD group (15.4\% vs. $23.4 \%, P=0.396)$, but these differences were not statistically significant. Notably, the en-bloc resection rate decreased drastically in tumors $\geq 20 \mathrm{~mm}$ for EMR $(17.4 \%$ vs. $75 \%, P<0.001)$ but not for ESD (27.6\% vs. $37.5 \%, P=0.587)$. Similarly, the differences in perforation ( $13.8 \%$ vs. $4.3 \%, P=0.097$ ) and bleeding rates $(10.3 \%$ vs. $15.9 \%, P=0.470)$ were not significant. Tumor size $\geq 20 \mathrm{~mm}$ was associated with a higher local recurrence (21.1\% vs. $6.5 \%, P=0.029)$. 

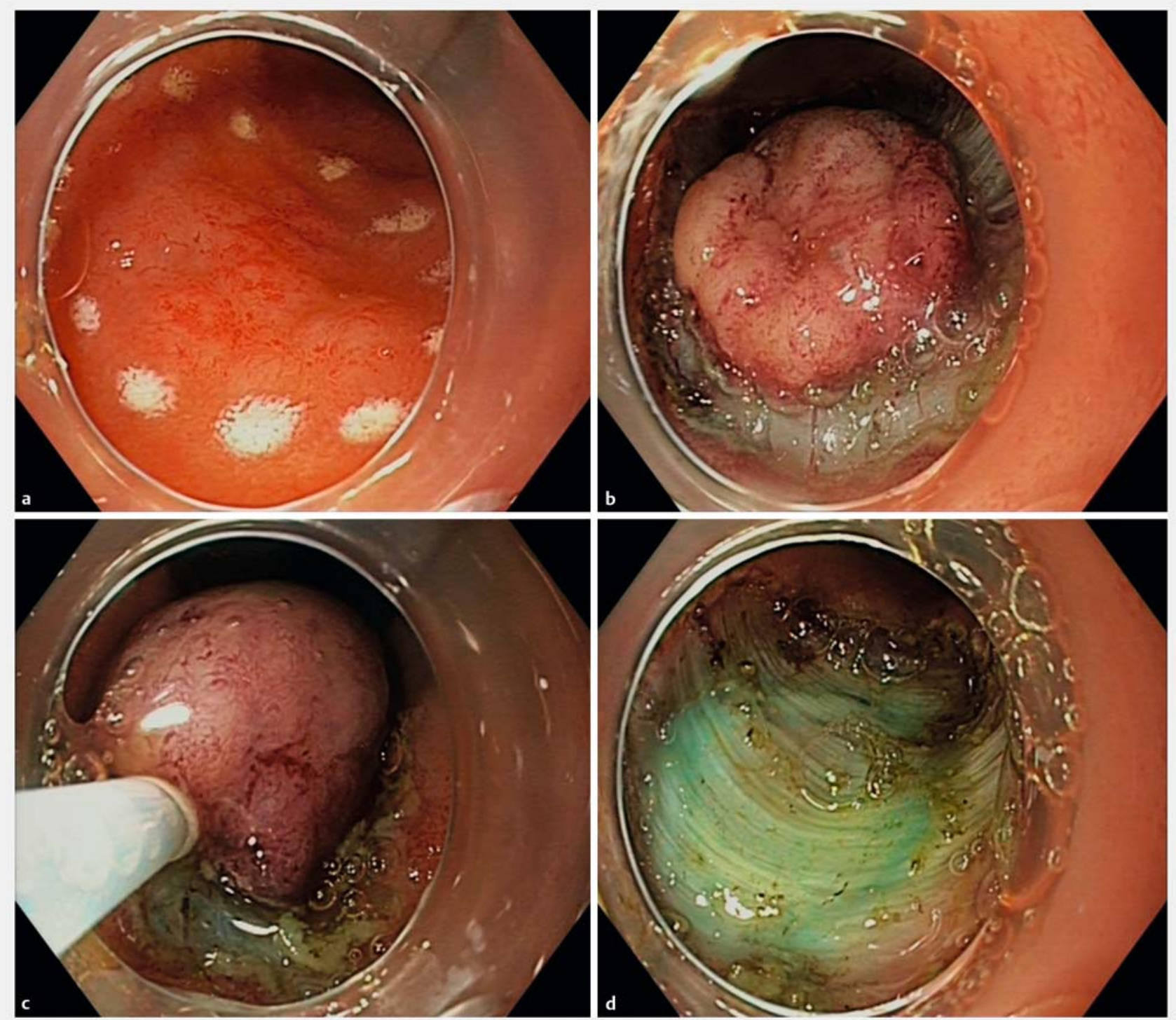

- Fig. 1 A 15-mm adenoma with high-grade dysplasia located in the bulb. Mucosal marking using a transparent hood (a). Circumferential endoscopic submucosal dissection by DualKnife (b). En-bloc hybrid resection using an asymmetric snare (c). Duodenal mucosal defect after resection (d).

\section{Discussion}

In the present series, we report on 166 NASDTs who underwent EMR or ESD with no differences in en-bloc and complete resection rates as well as local recurrence between both techniques. The en-bloc resection rates in the ESD group were comparable in large $(\geq 20 \mathrm{~mm})$ and small lesions, while this outcome decreased significantly in lesions resected by EMR. Overall, the en-bloc (10.6\% vs. $19.1 \%)$ and R0 resection rates (12.1\% vs. $18.6 \%$ ) were not statistically associated with lower recurrence, but the differences might be clinically relevant. This study demonstrated that ESD may have a higher risk of perforation compared to EMR.

There are few retrospective studies in the literature comparing ESD and EMR in duodenal lesions, and most of them have been reported by Japanese authors. In many cases, they included subepithelial and epithelial tumors all confounded [7-9] and considered patients with pedunculated lesions [10] or familial adenomatous polyposis [11]. Additionally, the criteria to choose ESD or EMR relies on the local experience and tumor characteristics; therefore, the populations are quite heterogeneous within different series. Furthermore, both groups may not be fully comparable, and the validity of the published results might be difficult to interpret and apply in a Western setting. In our series, we have performed ESD in large duodenal tumors, lesions suspected to be malignant, or with non-lifting sign. Indeed, half of the overall resected tumors were malignant and adenocarcinomas were confirmed to have a larger size and higher long-term recurrence rates. Thus, it is important to point out the malignant potential of these lesions, 
- Table 1 Outcomes of endoscopic resection procedures for 166 lesions in 150 patients with NASDTs.

\begin{tabular}{|c|c|c|c|}
\hline & $\operatorname{ESD}(n=37)$ & $\operatorname{EMR}(n=129)$ & $P$-value \\
\hline Age (median, range, years) & $64(44-83)$ & $66(31-82)$ & 0.187 \\
\hline $\begin{array}{l}\text { Tumor size (median, range, } \mathrm{mm} \text { ) } \\
\text { Size } \geq 20 \mathrm{~mm}\end{array}$ & $\begin{array}{l}25(12-50) \\
29(78.4 \%)\end{array}$ & $\begin{array}{l}20(5-50) \\
69(53.5 \%)\end{array}$ & $\begin{array}{l}0.010^{1} \\
0.007^{1}\end{array}$ \\
\hline Procedure time & $81(5-236)$ & $50(14-186)$ & $0.007^{1}$ \\
\hline En-bloc resection & $11(29.7 \%)$ & $57(44.2 \%)$ & 0.115 \\
\hline R0 resection ${ }^{2}$ & $7(19.4 \%)$ & $43(35.5 \%)$ & 0.069 \\
\hline \multicolumn{4}{|l|}{ Histology } \\
\hline - Adenoma, low-grade dysplasia & 22 & 57 & \\
\hline - Adenoma, high-grade dysplasia & 12 & 56 & \\
\hline - Adenocarcinoma & $2(5.6 \%)$ & $10(8.1 \%)$ & 0.607 \\
\hline - Other benign lesions & 1 & 6 & \\
\hline Closing of mucosal defect & $25(67.6 \%)$ & $88(68.2 \%)$ & 0.940 \\
\hline Perforation & $6(16.2 \%)$ & $3(2.3 \%)$ & $0.001^{1}$ \\
\hline - Intraoperative (major/minor) & $5(2 / 3)$ & $3(1 / 2)$ & \\
\hline - Delayed (major/minor) & $1(1 / 0)$ & 0 & \\
\hline Delayed bleeding & $3(9.3 \%)$ & $12(8.1 \%)$ & 0.823 \\
\hline Follow-up (median, range, months) & $4.5(2-84)$ & $10(2-125)$ & 0.278 \\
\hline Local recurrence ${ }^{3}$ & $5(14.7 \%)$ & $17(16.7 \%)$ & 0.788 \\
\hline \multicolumn{4}{|c|}{$\begin{array}{l}{ }^{1} \text { Statistically significant. } \\
{ }^{2} \text { En-bloc and R0 resection in } 157 \text { patients with adenoma or adenocarcinoma. } \\
{ }^{3} \text { Calculated for } 136 \text { patients with follow-up. }\end{array}$} \\
\hline
\end{tabular}

- Table 2 Characteristics of patients presenting with a duodenal perforation.

\begin{tabular}{|l|l|l|l|l|}
\hline $\begin{array}{l}\text { Spread- } \\
\text { ing }\end{array}$ & $\begin{array}{l}\text { Loca- } \\
\text { tion }\end{array}$ & Technique & $\begin{array}{c}\text { Type of } \\
\text { perfora- } \\
\text { tion }\end{array}$ & $\begin{array}{l}\text { Hospitali- } \\
\text { zation } \\
\text { (days) }\end{array}$ \\
\hline$<25 \%$ & D2 & Full ESD & Major & 4 \\
\hline $50 \%$ & D2 & Full ESD & Minor & 15 \\
\hline $50 \%$ & D3 & Hybrid ESD & Delayed & 10 \\
\hline $50 \%$ & D2 & Cap-EMR & Major & 7 \\
\hline $25 \%$ & D2 & Hybrid ESD 1 & Major & 2 \\
\hline $66 \%$ & D2 & EMR & Minor & 2 \\
\hline $50 \%$ & D2 & Hybrid ESD 2 & Minor & 2 \\
\hline $75 \%$ & D2 & EMR & Minor & 3 \\
\hline$<25 \%$ & Bulb & Hybrid ESD2 & Minor & 3 \\
\hline 1 The perforation occurred during snare resection. & \\
\hline 2 The perforation occurred during the ESD procedure. & \\
\hline
\end{tabular}

where complete endoscopic resection might avoid major surgery.

This study represents the second series of duodenal ESD in a Western setting where the ESD is not as developed as in the Asian world; however, this is one of the largest reports published to date. Naturally, en-bloc resection is mainly related to tumor size. According to Basford et al. [5], we have performed a hybrid ESD in patients with larger lesions compared to those resected by piecemeal EMR. The few studies to date regarding ESD in duodenal tumors included selected patients with heterogeneous mean sizes $(10-22 \mathrm{~mm})[11,12]$ and locations. Indeed, Hoteya et al. [6] reported a higher complete resection rate in large NASDTs (mean size: $31.3 \mathrm{~mm}$ ) compared to small lesions (mean size: $11.6 \mathrm{~mm}$ ) resected by ESD. Despite this procedure being technically challenging, the en-bloc resection rates in Asian series have been reported as above 90\% [13]. However, the included number of lesions is less than 10 in most of these reports $[7,9,11,12,14,15]$, and the impact of en-bloc resection in local recurrence is still unclear. In our series, we considered 37 NASDTs who underwent ESD. The enbloc resection rate was lower $(29.7 \%)$ than previously described and lower compared to the EMR group, probably because of the higher tumor size (median: $25 \mathrm{~mm}$ ) and the hybrid ESD approach in $78.4 \%$ of cases. Similarly, the complete resec- 
tion rate was higher in the EMR group and not associated with recurrence.

Although overall recurrence following EMR has been described up to $37 \%$ and associated with tumor size [16], endoscopic resection of residual adenoma has been described to be successful $[17,18]$. Navaneethan et al. [19], in a systematic review and meta-analysis on duodenal EMR of 440 patients from 14 studies, concluded a recurrence rate of $15 \%$, with no association based on the initial type of resection (en-bloc or piecemeal). Similarly, in our series, the recurrence was $16.7 \%$ in the EMR group, and no association of recurrence was observed with the endoscopic technique, but only with a larger tumor size. We have also considered a 20 -mm threshold as proposed by other authors $[8,11]$, and the results were similar to the overall population for the main outcomes. Thus, these outcomes were probably higher in the EMR group because of the smaller tumor size and ESD learning curve, but this difference was much lower in local recurrence rates.

Safety is a main concern in ESD and particularly in duodenal ESD where the benefits of the technique remain unknown. Although one may argue that the higher risk of perforation in this location is also because of the poor development of the technique at the early beginning of the learning curve, most studies reporting on duodenal ESD have been carried out by experienced endoscopists in referral centers. Indeed, the risk of perforation is a major issue and occurs in $7-39 \%$ of cases [ 6 , $20,21]$. Conversely, other authors $[19,22,23]$ reported a $0-$ $2.8 \%$ perforation rate by using the EMR technique, concluding that this approach is safe even in large spreading lesions [24]. Recently, underwater EMR has been also described as a successful technique in small adenomas with an en-bloc resection rate of $87 \%$ [25]. Our overall perforation rate in lesions resected using a DualKnife was $16.2 \%$. There were probably other factors that led to this complication, as the limited scope maneuverability, fibrosis, non-lifting, and previous polypectomy, but they were difficult to assess retrospectively. However, the perforation was identified during the procedure and managed endoscopically in all but 1 patient who presented with a delayed perforation. This very low rate of delayed perforations may be due to the careful inspection of the mucosal defect after the procedure and systematic closure, sometimes using different hoods and contrast under radiological control. Similarly, delayed bleeding was much more frequent in lesions without prophylactic closure ( $18.9 \%$ vs. $4.4 \%$ ).

This study has several limitations. Different factors such as tumor morphological type and scope maneuverability may have influenced the choice between EMR and ESD. The different operators during a wide inclusion period and the consequent learning curve may have influenced the outcomes. Additionally, the retrospective design and the lost to follow-up of $18 \%$ may have underestimated the overall local recurrence.

In conclusion, ESD may be an alternative to EMR and surgery in selected NASDTs, such as large duodenal tumors where EMR achieves lower en-bloc resection rates and the local recurrence rate may be higher. However, this technique may have a higher risk of perforations. Moreover, high-grade dysplasia and invasive cancer are frequent in NASDTs and should be suspected in large lesions. Prospective studies including a higher number of patients with similar tumor characteristics are needed to clarify the association between the endoscopic features and local recurrence in this location, where it is very difficult to assess the malignancy preoperatively.

\section{Competing interests}

None

\section{References}

[1] Deprez PH, Bergman JJ, Meisner S et al. Current practice with endoscopic submucosal dissection in Europe: position statement from a panel of experts. Endoscopy 2010; 42: $853-858$

[2] Fuccio L, Hassan C, Ponchon T et al. Clinical outcomes after endoscopic submucosal dissection for colorectal neoplasia: a systematic review and meta-analysis. Gastrointest Endosc 2017; 86: 74-86

[3] Kikuchi D, Hoteya S, lizuka T et al. Diagnostic algorithm of magnifying endoscopy with narrow band imaging for superficial non-ampullary duodenal epithelial tumors. Dig Endosc 2014; 26: 16-22

[4] Pimentel-Nunes P, Dinis-Ribeiro M, Ponchon T et al. Endoscopic submucosal dissection: European Society of Gastrointestinal Endoscopy (ESGE) Guideline. Endoscopy 2015; 47: 829-854

[5] Basford PJ, George R, Nixon E et al. Endoscopic resection of sporadic duodenal adenomas: comparison of endoscopic mucosal resection (EMR) with hybrid endoscopic submucosal dissection (ESD) techniques and the risks of late delayed bleeding. Surg Endosc 2014; 28 : $1594-1600$

[6] Hoteya S, Furuhata T, Takahito T et al. Endoscopic submucosal dissection and endoscopic mucosal resection for non-ampullary superficial duodenal tumor. Digestion 2017; 95: 36-42

[7] Park SM, Ham JH, Kim BW et al. Feasibility of endoscopic resection for sessile nonampullary duodenal tumors: a multicenter retrospective study. Gastroenterol Res Pract 2015; 2015: 692492

[8] Matsumoto S, Yoshida Y. Selection of appropriate endoscopic therapies for duodenal tumors: an open-label study, single-center experience. World J Gastroenterol 2014; 20: 8624-8630

[9] Teoh AY, Chiu PW, Chan SY et al. Hospital Authority audit of the outcome of endoscopic resection of superficial upper gastro-intestinal lesions in Hong Kong. Hong Kong Med J 2015; 21: 224-231

[10] Kakushima N, Ono H, Takao T et al. Method and timing of resection of superficial non-ampullary duodenal epithelial tumors. Dig Endosc 2014; 26 : $35-40$

[11] Nonaka S, Oda I, Tada K et al. Clinical outcome of endoscopic resection for nonampullary duodenal tumors. Endoscopy 2015; 47: 129 135

[12] Endo M, Abiko Y, Oana $S$ et al. Usefulness of endoscopic treatment for duodenal adenoma. Dig Endosc 2010; 22: 360-365

[13] Yamamoto Y, Yoshizawa N, Tomida H et al. Therapeutic outcomes of endoscopic resection for superficial non-ampullary duodenal tumor. Dig Endosc 2014; 26: 50 - 56

[14] Seo JY, Hong S], Han JP et al. Usefulness and safety of endoscopic treatment for nonampullary duodenal adenoma and adenocarcinoma. J Gastroenterol Hepatol 2014; 29: $1692-1698$

[15] Zhong YS, Shi Q, Wu HF et al. Endoscopic resection for the treatment of duodenal Brunner's adenoma. J Laparoendosc Adv Surg Tech A 2012; 22: 904-909 
[16] Abbass R, Rigaux J, Al-Kawas FH. Nonampullary duodenal polyps: characteristics and endoscopic management. Gastrointest Endosc 2010; 71: $754-759$

[17] Alexander S, Bourke M], Williams S] et al. EMR of large, sessile, sporadic nonampullary duodenal adenomas: technical aspects and long-term outcome (with videos). Gastrointest Endosc 2009; 69: $66-73$

[18] Apel D, Jakobs R, Spiethoff A et al. Follow-up after endoscopic snare resection of duodenal adenomas. Endoscopy 2005; 37: 444-448

[19] Navaneethan U, Hasan MK, Lourdusamy V et al. Efficacy and safety of endoscopic mucosal resection of non-ampullary duodenal polyps: a systematic review. Endosc Int Open 2016; 4: E699-E708

[20] Honda T, Yamamoto H, Osawa $\mathrm{H}$ et al. Endoscopic submucosal dissection for superficial duodenal neoplasms. Dig Endosc 2009; 21: $270-274$

[21] Hoteya S, Yahagi N, lizuka T et al. Endoscopic submucosal dissection for nonampullary large superficial adenocarcinoma/adenoma of the duodenum: feasibility and long-term outcomes. Endosc Int Open 2013; 1: E2 -E7

[22] Tomizawa Y, Ginsberg GG. Clinical outcome of endoscopic mucosal resection of sporadic, nonampullary duodenal adenoma: a 10-year retrospective. Gastrointest Endosc 2018: doi:10.1016/j. gie.2017.12.026 [Epub ahead of print]

[23] Klein A, Nayyar D, Bahin FF et al. Endoscopic mucosal resection of large and giant lateral spreading lesions of the duodenum: success, adverse events, and long-term outcomes. Gastrointest Endosc 2016; 84: $688-696$

[24] Singh A, Siddiqui UD, Konda V] et al. Safety and efficacy of EMR for sporadic, nonampullary duodenal adenomas: a single U.S. center experience (with video). Gastrointest Endosc 2016; 84: 700 - 708

[25] Yamasaki Y, Uedo N, Takeuchi Y. Underwater endoscopic mucosal resection for superficial nonampullary duodenal adenomas. Endoscopy 2018; 50: $154-158$ 